1 IMAGING CARDIOVASCULAR FEATURES OF A FAMILY WITH TYPE 4 LOEYS-DIETZ SYNDROME

Irilda Budaj, Prity Gupta, Anand Saggar, Christopher Nienaber, Deborah Morris-Rosendahl, Saeed Mirsadraee. Royal Brompton and Harefield NHS Foundation Trust (London, UK)

10.1136/heartjnl-2019-BSCI.1

Introduction Loeys-Dietz syndrome (LDS) is an inherited autosomal dominant disorder caused by pathogenic variants in the genes involved in TGF $\beta$ signaling. There is a wide phenotypic spectrum of cardiovascular, skeletal, craniofacial and cutaneous manifestations. The hallmark of this rare connective tissue disorder is progressive aortic and peripheral arterial aneurysmal disease leading to acute events. We evaluated the range of cardiovascular abnormalities in a family with type 4 LDS. The background literature data and screening strategy is discussed.

Methods We retrospectively studied the radiological cardiovascular findings in 23 members of a family with features of LDS who were monitored with echocardiogram. 17 were investigated with MRI and 9 with CT. The head and neck arteries, the aorta and its visceral branches, iliac and femoral arteries were assessed. Size of the vessels, tortuosity, length and presence and the onset of arterial dissection were investigated.

Results 11/23 patients revealed arterial abnormalities, all with TGFB2 variant. Two patients had type-A dissection at the age of 48 and 49 , one treated surgically and the other medically. Three patients with aortic root dilatation underwent preventive personalized external aortic root support procedure (PEARS). The other abnormalities included: mild aortic root dilatation (3), infrarenal aortic aneurysm (1), tortuosity of carotid (2) and vertebral arteries (1), aneurysmal iliac arteries (1), dilated coeliac trunk and superior mesenteric arteries (1).

Conclusion Vascular abnormalities are common in patients with familial history of LDS and can predispose to potentially fatal events in later life. Assessing for cardiovascular risk in LDS is crucial.

\section{RELATIVE VALUE OF CARDIAC MRI AND FDG-PET IN TREATMENT FOLLOW-UP FOR CARDIAC SARCOIDOSIS}

Richard Coulden, Emer Sonnex, Jonathan Abele. University of Alberta Hospital, Edmonton, $A B$, Canada

\subsection{6/heartjnl-2019-BSCI.2}

Introduction ${ }^{18}$ FDG PET-CT and cardiac MR (CMR) are described in the diagnosis of cardiac sarcoidosis (CS), each identifying different aspects of the disease. We compared PETCT and CMR in the follow-up of patients with suspected CS. Methods 31 patients with proven extra-cardiac sarcoidosis and possible CS were identified from the local sarcoidosis registry. All had combined PET-CT and CMR on two occasions. Selected patients had not received immunosuppressants for at least 6 months before the initial combined study. 22 patients were treated with immunosuppressants for 3 months or longer. In 9, the clinician chose not to treat or the patient refused treatment. Follow-up PET-CT and CMR were done 102-770 days later (median 228).

Results Significant myocardial FDG uptake was shown on visit 1 in 17 treated patients (myocardial SUVmax >3.6). Myocardial SUVmax fell significantly on follow-up $(p<0.01)$ and was matched falls in FDG avid lung and node disease. Treated patients also showed improvement in left ventricular ejection fraction $(\mathrm{LVEF})(\mathrm{p}=0.03) .14$ treated patients had late gadolinium enhancement (LGE) which was not necessarily matched by FDG. This was unchanged by visual assessment on followup. In 9 untreated patients (4 with FDG avid myocardium and 5 with LGE), there was no change in myocardial FDG, LGE or LVEF.

Conclusion Myocardial FDG uptake in CS represents active inflammation. When treated, this resolved or regressed on follow up with an improvement in LVEF and FDG avid thoracic disease. There was no change in extent of LGE. Untreated patients showed no change.

\section{USPIO-ENHANCED MAGNETIC RESONANCE CORONARY ANGIOGRAPHY COMPARED TO COMPUTED TOMOGRAPHY CORONARY ANGIOGRAPHY}

Marwa Daghem, Rishi Ramaesh, Gillian Macnaught, Marc R Dweck, Scott Semple, Edwin JR van Beek, David E Newby, Michelle C Williams. University of Edinurgh, Edinburgh, UK

\subsection{6/heartjnl-2019-BSCI.3}

Background Recent concerns regarding gadolinium has led to the investigation of alternatives for magnetic resonance coronary angiography (MRCA) including non-contrast imaging and contrast imaging with ultrasmall superparamagnetic particles of iron oxide (USPIO).

Methods Seven patients underwent USPIO-enhanced MRCA using a 3 Tesla PET/MRI scanner and computed tomography (CT) coronary angiography (CTCA) using a 128-multidetector computed tomography (CT) scanner. MRCA was performed using FLASH magnetic resonance angiography sequences with 3 to $4 \mathrm{mg} / \mathrm{kg}$ of intravenous ferumoxytol. Image analysis was performed by two observers blinded to other imaging. Per segment image quality was rated on a 4-point scale. Per vessel coronary assessment was performed, with segments classified as less than or greater than $50 \%$ luminal diameter.

Results 99 segments in 7 patients were assessed. Diagnostic image quality was observed in $80 \%$ (79/99) of segments with USPIO-enhanced MRCA compared to $99 \%$ (98/99) with CCTA. 85 segments in 21 vessels were available for the assessment of stenoses. On CCTA, 12 segments in 8 vessels had one or more $>50 \%$ stenosis. Per vessel diagnostic accuracy of USPIO-enhanced MRCA was good with sensitivity $63 \%$, specificity $77 \%$, positive predive value (PPV) 63\%, negative predictive value (NPV) $77 \%$, and accuracy $71 \%$, and was better for proximal compared to distal segments.

Conclusion USPIO-enhanced MRCA has a good image quality for the identification of vessel segments and stenoses, particularly in proximal segments.

\section{STUDYING CONTRAST ADMINISTRATION IN THE FONTAN CIRCULATION USING MR TIME RESOLVED ANGIOGRAPHY}

${ }^{1}$ Laura Duerden, ${ }^{2}$ Mark Hamilton. ${ }^{1}$ Royal United Hospitals Bath NHS Foundation Trust, Bath, UK; ${ }^{2}$ Bristol Royal Infirmary, Universities Hospital Bristol NHS Foundation Trust, Bristol, UK

\subsection{6/heartjnl-2019-BSCl.4}

Introduction When patients with Fontan circulation require CT imaging, there are significant challenges in achieving adequate contrast opacification due to altered anatomical 
connections. This study uses MR time resolved angiography to examine contrast circulation in a cohort of patients with Fontan circulation to optimise contrast injection protocol for CT. Methods Time to peak signal intensity was recorded using regions of interest on the aorta, the pulmonary arteries and the Fontan conduit on MR TWIST angiography images. Patients were stratified into groups according to ejection fraction, global longitudinal strain, indexed stroke volume and cardiac index to examine the effect on time to peak signal intensity.

Results 35 patients were included in the study. Mean time to peak contrast enhancement was $31 \mathrm{~s}$ in the thoracic aorta, 46s in the right pulmonary artery, 41s in the left pulmonary artery and $55 \mathrm{~s}$ in the Fontan conduit. Cardiac performance shows little relationship to peak vascular enhancement whether measured by ejection fraction, global longitudinal strain, stroke volume index and cardiac index.

Discussion This MRI data suggest that optimal timing for a single phase examination to show all the major vessels is around 55 seconds following start of contrast injection. In TWIST MR angiography the IV bolus is 4-5 seconds long. A longer bolus is required for CTA, around 20s, suggesting an additional delay will be required. Further work will be done to see is a single phase examination at 70 seconds is an ideal initial strategy, with targeted further imaging if unsuccessful.

\section{THORACIC AORTA AND PULMONARY ARTERY DIMENSIONS IN PATIENTS UNDERGOING ECG-GATED COMPUTED TOMOGRAPHY ANGIOGRAPHY}

${ }^{1}$ Andrew Gentle, ${ }^{1,2}$ Michelle C Williams, ${ }^{1,2}$ David E Newby, ${ }^{3,4}$ Giles Roditi. ' University of Edinburgh/British Heart Foundation Centre for Cardiovascular Science, Edinburgh, UK; ${ }^{2}$ Edinburgh Imaging facility QMRI, University of Edinburgh, Edinburgh, UK; ${ }^{3}$ Glasgow Clinical Research Imaging Facility, Queen Elizabeth University Hospital, Glasgow, UK; ${ }^{4}$ Glasgow University, Glasgow, UK

\subsection{6/heartjnl-2019-BSCI.5}

Introduction Normal values for vascular dimensions are derived from echocardiography or magnetic resonance imaging (MRI), with limited data available on electrocardiogram (ECG)-gated computed tomography (CT) angiography. This abstract assesses aortic and pulmonary artery diameters and the factors affecting them, in patients undergoing CT as part of the SCOT-HEART (Scottish COmputed Tomography of the HEART) trial.

Methods Vessel diameters were measured on CT coronary angiography (CTCA) in six locations: annulus, sinus of Valsalva (SOV), sinotubular junction (STJ), ascending and descending aorta at right pulmonary artery level, and main pulmonary artery (MPA). The SCOT-HEART database provided demographics and cardiovascular risk factor information.

Results Images of 1000 patients (mean age 58.9, 58\% male) were assessed. Amongst patients with normal coronary arteries and no history of hypertension, mean dimensions were: annulus short-axis $19 \pm 2 \mathrm{~mm}$, annulus long-axis $27 \pm 3$ $\mathrm{mm}$, SOV $31 \pm 4 \mathrm{~mm}$, STJ $27 \pm 3 \mathrm{~mm}$, ascending aorta $30 \pm 4$ $\mathrm{mm}$, descending aorta $22 \pm 2 \mathrm{~mm}$, and MPA $23 \pm 3 \mathrm{~mm}$. Male sex, height, and body surface area (BSA) correlated with increases in all dimensions $(\mathrm{p}<0.001)$. Age correlated with aortic dimensions only $(\mathrm{p}<0.03)$. Patients with normal coronary arteries on CTCA had smaller aortic dimensions $(\mathrm{p}<0.001)$. Patients with hypertension had larger MPA and aortic dimensions $(p<0.02)$, apart from at the annulus. Sex and BSA were independent predictors of all dimensions. Hypertension was an independent predictor of some aortic dimensions. Age was an independent predictor of all aortic dimensions, except the annulus.

Conclusion Aortic and pulmonary dimensions can be assessed on CTCA, with sex and BSA having an important impact on normal dimensions.

\section{COMPARISON CTCA DOSE AUDIT BETWEEN INITIAL SERVICE DEVELOPMENT AND SUBSEQUENT SERVICE ESTABLISHMENT PHASE FOR A DGH CTCA SERVICE}

Vilim Kalamar, Peter Chapman, Julian Elford, Jade Fleet, Alison Wright, Zoe Crawley. Department of Radiology Hampshire Hospitals NHS Foundation Trust, Winchester, UK

\subsection{6/heartjnl-2019-BSCI.6}

Introduction The delivery of computed tomography coronary angiography (CTCA) services is variable but underprovided across the UK. Hampshire hospitals foundation trust (HHFT) is trying to contribute to this necessary national increase in CTCA provision. The aim of this poster presentation is presentation of our initial ionizing radiation dose audits for governance and to share issues we faced locally which might be relevant to other sites starting similar services.

Methods Retrospective analysis of all patients attending HHFT for CTCA at HHFT was obtained by interrogation of the Computerized Radiology Information System (CRIS) database between 2013 and April 2018, then re-audited between May 2018 and February 2019.The total Dose-Length Product (DLP) units ( $\mathrm{mGycm}$ ) and heart rate at time of acquisition were identified allowing comparison between the initial service development \& second service establishment phase.

Results The median total DLP was 113.11 during initial service development (January 2013 - April 2018 phase). The median total DLP was 192.91 during service establishment phase (May 2018 - February 2019) Median DLP became higher in establishment phase.

Conclusion Initial success of the service along with initial tight patient selection led to a lower first round DLP. Subsequent appointment of an Electrophysiology Cardiology Consultant and increasing awareness of service led to evolving referral patterns with more atrial fibrillation (AF) ablation scans requested. Identifying this in the second round of audit has led to discussions with referrers \& establishment of routine DLP recording onto CRIS

\section{RADIATION DOSES OF CT CORONARY ANGIOGRAMS DONE AT SOUTHEND UNIVERSITY HOSPITAL; ONE YEAR AFTER THE 2017 NATIONAL SURVEY}

Mohammad Ali Khan, Harith Adil Al-Qaysi. Southend University Hospital, UK

\subsection{6/heartjnl-2019-BSCI.7}

Introduction In 2018, Southend University Hospital performed 846 CT coronary angiograms. We participated in the 2017 Prospective National Survey of coronary CT radiation. Our median radiation dose was $194 \mathrm{mGycm}$ compared to the national median of $209 \mathrm{mGycm}$. After the audit, 64 slice scanners with improved low contrast detectability were installed. These were powered by Adaptive Statistical Iterative 